\title{
Effects of an Inhomogeneous Temperature Distribution at the CMB on Polarity Reversals of the Earth's Magnetic Field
}

\author{
Y. Honkura, T. IIjima, M. Matsushima, and Y. Tanahashi \\ Department of Earth and Planetary Sciences, Tokyo Institute of Technology, \\ 2-12-1 Ookayama, Tokyo 152, Japan
}

(Received January 5, 1993; Revised September 20, 1993; Accepted October 19, 1993)

\begin{abstract}
Time evolution of the magnetic field is examined numerically for some models of thermally driven MHD dynamo in a spherical shell. Special attention is paid to whether the polarity of the dipole magnetic field, in the models, reverses its sign during time evolution. Here we present the results for two typical models. In one model, the temperature is held constant at both boundaries of a spherical shell, whereas in the other, the distribution of temperature is represented by the degree 2 and order 2 constituent of spherical harmonics. The former shows an oscillatory variation and hence the polarity is reversed rather periodically. The latter results in a rather stationary state and the magnetic field tends to stay at one polarity state. These numerical results imply that the thermal interaction between the core and the mantle can control the frequency of reversal.
\end{abstract}

\section{Introduction}

Recent progress in studies of paleosecular variation recorded in sediments provoked an exciting argument about the mechanism of polarity reversal of the Earth's magnetic field. The discussion is based usually on the behavior of VGP (virtual geomagnetic pole) during transitional stages (e.g. Hoffman, 1992; LAJ et al., 1992). Progress in theoretical studies, however, is rather slow mainly because a purely theoretical treatment of a non-linear MHD dynamo problem is extremely difficult and also numerical computation requires huge memory capacity and computation time. Hence some dynamo models avoid such difficulty and rely on the so-called $\alpha$-effect, and polarity reversals have been shown to occur in such models (e.g. OLSON and HAGEE, 1990).

How the polarity of the Earth's magnetic field is reversed has been a long lasting problem and the recent observational and theoretical studies seem to provide a realistic insight into this problem. In fact, some physical mechanisms have been proposed for polarity reversals (e.g. GubBINS, 1987). In particular, the thermal interaction between the core and the base of the lower mantle, usually referred to as the $\mathrm{D}^{\prime \prime}$ layer, has been considered as a process greatly affecting the Earth's magnetic field (Jones, 1977; Bloxham and GubBins, 1987; GubBins, 1988).

However, the role of such a thermal interaction on the magnetic field seems to be different from one model to another. In one model, the interaction is supposed to result in a polarity reversal (e.g. GubBins, 1987). In another model, the interaction is supposed to control the frequency of reversal (e.g. JonEs, 1977).

We have been continuing calculations of time evolution of the magnetic field for models of thermally driven MHD dynamo in a spherical shell and found that the dipole field can reverse its polarity in some models (HoNkURA et al., 1992). The behavior turned out to be similar to that in some simple disk dynamo models (Shimizu and HonkURA, 1985; HonkurA and MATSUSHIMA, 1988). This result suggests that the Earth's magnetic field reverses its polarity spontaneously, as was first demonstrated in the Rikitake coupled disk dynamo model (RIKITAKE, 1958; AlLAN, 1962). 
We are now confronted two different ideas as to the mechanism of polarity reversal. Which would be the case, spontaneous reversal or triggered reversal? In addition, the role of the thermal interaction between the core and the mantle complicates the situation. In this paper, we attempt a unified view for these problems on the basis of our numerical calculations for some simple MHD dynamo models. The hypothesis which we try to test in this paper is that the magnetic field reverses its polarity spontaneously and probably chaotically, but the long-term trend in the frequency of reversal is controlled by the thermal interaction between the core and the mantle.

\section{A Homogeneous Model}

In our previous paper (HONKURA et al., 1992), we presented our formulation of numerical computation of MHD dynamo in a spherical shell, together with our definition of some dimensionless numbers; the thickness of the shell $(d)$ is taken as the characteristic length scale, the typical time for thermal diffusion $\left(d^{2} / \kappa\right)$ as the characteristic time, the temperature difference between the top and the bottom of the shell $(\Delta T)$ as the temperature, and $(\nu \kappa \rho \mu)^{1 / 2} / d$ as the magnetic field. The ratio of the inner to outer radii is taken as 0.4 . We expanded the velocity,
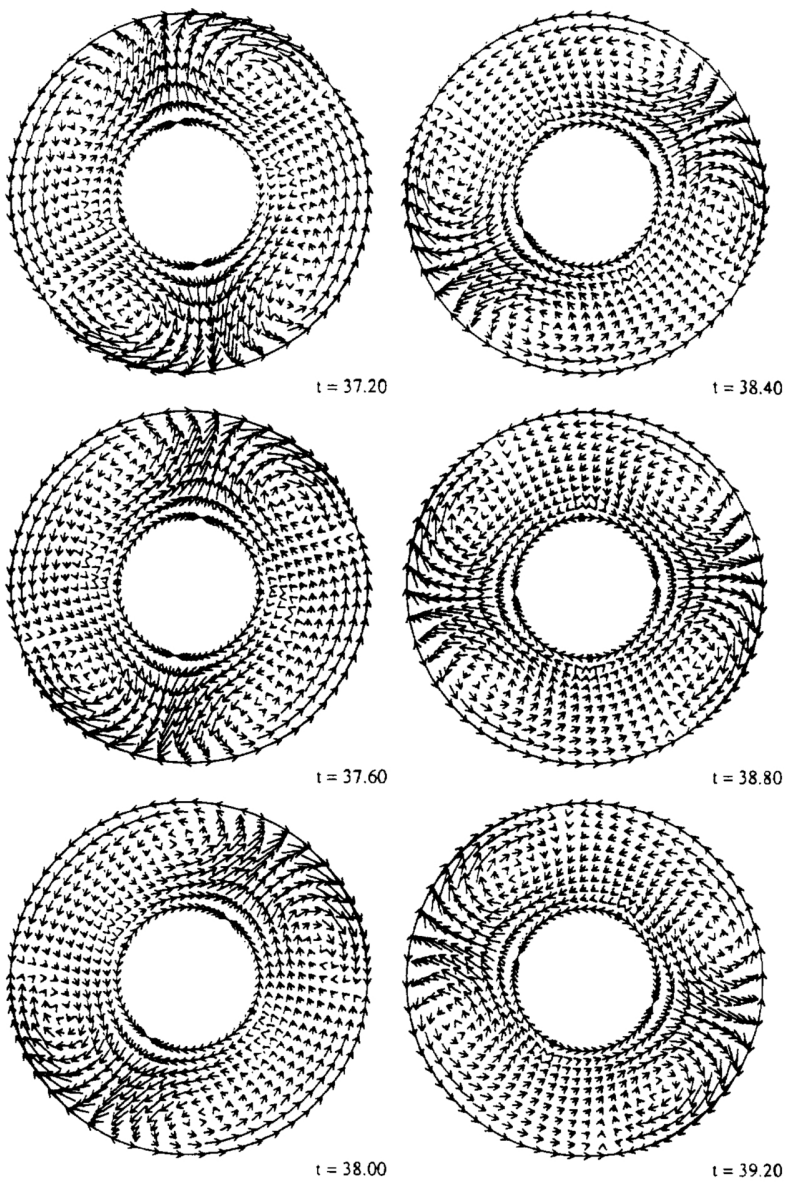

Fig. 1. Pattern of convective motion in the spherical shell. Arrows indicate the velocity field on the equatorial plane viewed from the north pole side. 

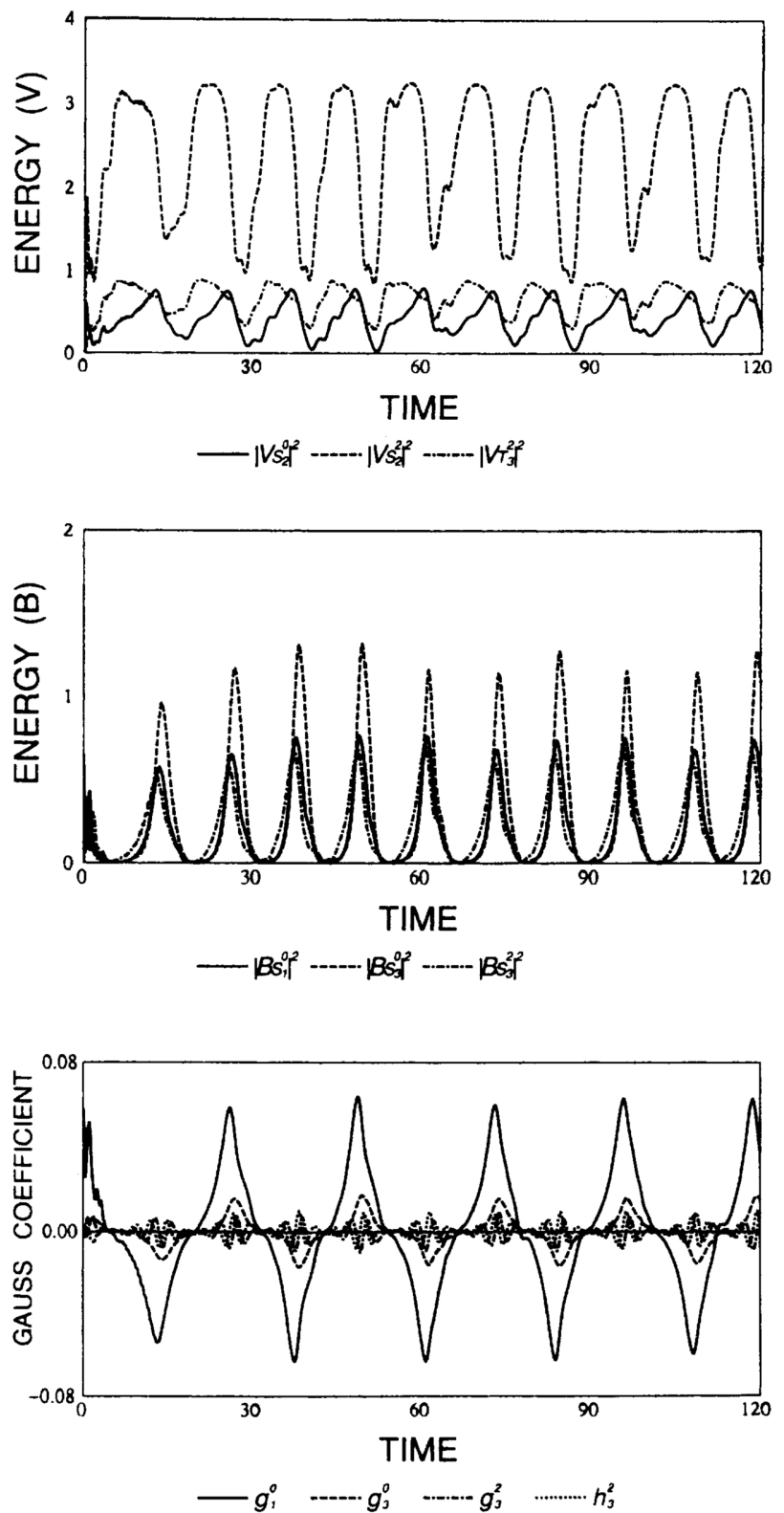

Fig. 2. Time evolution of the dominant constituents of the velocity and the magnetic fields in terms of their energy, and time evolution of the Gauss coefficients. Homogeneous model $(\operatorname{Ra}=900, \operatorname{Pr}=1, T a=100$, $P m=30$ ). 
the temperature, and the magnetic fields into a set of series of spherical harmonics for $\theta$ and $\phi$, and trigonometric functions for $r$. Then the boundary conditions are all satisfied, implicitly in the expression except for the poloidal magnetic field constituents; in particular, the temperature is held constant at both boundaries of the spherical shell. In this method, therefore, no numerical instability arises during time evolution unless the time step is taken too large.

The only problem is that we have been unable to check how stable the derived time evolution would be with respect to the truncation level in a set of series. Our preliminary calculations indicate that the velocity field changes little between the cases $L=4$ and $L=6$ in spherical harmonics and also between the cases $N=4$ and $N=6$ in trigonometric functions. Therefore we set the truncation level at $L=N=4$ and examined time evolution of the velocity field, as shown in Fig. 1. Such rapid convergence is due to small values of dimensionless numbers $(\operatorname{Pr}=1$, $R a=900, T a=100$; see HoNkURA et al. (1992)), and hence if, for instance, the Taylor number is increased, similar convergence is unlikely to be guaranteed, as suggested in ZHANG and BUSSE (1987).

With $L$ and $N$ fixed at 4 , we examined time evolution of not only the velocity and the temperature fields but also the magnetic field, although the convergence has not been fully examined in this case. Figure 2 shows time evolution of the magnetic field in terms of the Gauss coefficients. Since we have considered the so-called dipole family only, with the velocity field symmetric with respect to the equatorial plane, the terms of $l+m=$ odd only appear and the dipole term $\left(g_{1}^{0}\right)$ turned out to be dominant in the magnetic potential expression for the Earth's surface.

This figure clearly indicates that the dipole field reverses its polarity spontaneously. It should be remembered here that no specific boundary conditions are imposed; the temperature is just held constant. Such a behavior may turn out to be an artifact arising from the insufficient truncation level. Nonetheless, this result would be of significance if the comparison is made with the results at the same truncation level for other models with different boundary conditions.

In particular, our present concern is whether a polarity reversal is controlled by a specific boundary condition at the CMB (core-mantle boundary) or whether it occurs spontaneously as a result of non-linear interaction involved in dynamo processes within the core. The result shown in Fig. 2 indicates that the latter case is possible. In order to investigate the former case, we examine, in the next section, time evolution of the magnetic field for an intentionally imposed inhomogeneous boundary condition at the outer boundary. Hereafter, the model of HonkURA et al. (1992) is referred to as a homogeneous model and the one with an inhomogeneous boundary condition will be called an inhomogeneous model.

\section{An Inhomogeneous Model}

Since the temperature is held constant at the boundaries in the homogeneous model, a natural selection for an inhomogeneous model would be the condition of variable temperature at the outer boundary. If the relevance of a model to the real Earth is to be pursued, this model may not be appropriate, because the temperature gradient should be very small in the Earth's core and the temperature varies little, laterally, at the outer-most core (BLOXHAM and JACKSON, 1990).

On the other hand, dynamical behavior might be different between the boundary condition of homogeneous temperature and the condition of homogeneous temperature gradient, and hence homogeneous and inhomogeneous models cannot be compared directly if a homogeneous temperature condition is used for a homogeneous model and an inhomogeneous temperature gradient condition is used for an inhomogeneous model. For this reason, we reserve the condition of temperature itself and examine the effect of an inhomogeneous temperature distribution, with the comparison in mind with the homogeneous case. A similar problem was analyzed in more detail by ZHANG and GuBBins $(1992,1993)$ for dynamical aspects only. 
What type of inhomogeneity should we impose at the outer boundary? Here we should remember that the dominant convective motion is the constituent of $(l, m)=(2,2)$, as clearly shown in Fig. 1. Moreover, this pattern drifts towards the west. These two seem to be the most fundamental dynamical properties of the homogeneous model. In a typical inhomogeneous model, these properties should be perturbed. We therefore select the inhomogeneity represented by $(l, m)=(2,2)$.

The modification for this case is easy. The basic unperturbed temperature is the superposition of $T_{0}$ in HONKURA et al. (1992) and $T_{1}$ representing the inhomogeneous temperature distribution, given as

$$
\begin{gathered}
T_{0}=C_{1}+\frac{C_{2}}{r}, \\
T_{1}=\left(C_{3} r^{2}+\frac{C_{4}}{r^{3}}\right) P_{2}^{2}(\cos \theta) \cos 2 \phi,
\end{gathered}
$$

where $C_{1}, C_{2}, C_{3}$, and $C_{4}$ are constants. $C_{1}$ is arbitrary, but $C_{2}, C_{3}$, and $C_{4}$ must satisfy

$$
\begin{gathered}
C_{2}\left(\frac{1}{r_{i}}-\frac{1}{r_{o}}\right)=1, \\
C_{3} r_{o}^{2}+\frac{C_{4}}{r_{o}^{3}}=A, \\
C_{3} r_{i}^{2}+\frac{C_{4}}{r_{i}^{3}}=0,
\end{gathered}
$$

where $r_{i}$ and $r_{o}$ denote the radii of the inner and outer boundaries, and $A$ represents the amplitude of the temperature variation at the outer boundary.

Figure 3 shows the temperature $T_{1}$ at the outer boundary. In this study, the amplitude, denoted by $A$ in the above expression, is also an important parameter, since it represents the degree of influence of the outer boundary. In fact, as we increase the amplitude, a rather periodic state as shown in HONKURA et al. (1992) is modified gradually and shifts eventually to another stable state as will be shown below.

We now show the results for $A=0.03$. In this case, however, the magnetic Prandtl number was reduced to 10 in order to keep the parameter values slightly above the critical ones; the other parameter values are unchanged. Figure 4 shows the pattern of convective motion on the equatorial plane. The most striking nature is that the pattern drifts neither westwards any longer nor eastwards.

As shown in Fig. 4, the upwellings are shifted by a few tens of degrees from the high temperature areas. This nature is rather contrary to the feature expected from the thermal wind

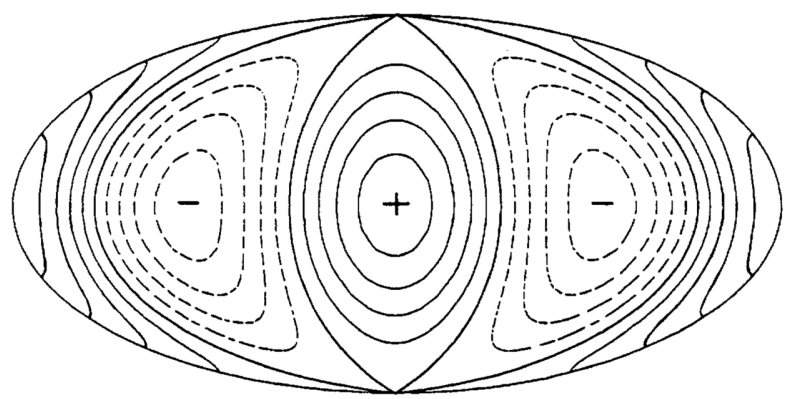

Fig. 3. Distribution of the temperature at the outer boundary of the spherical shell. 

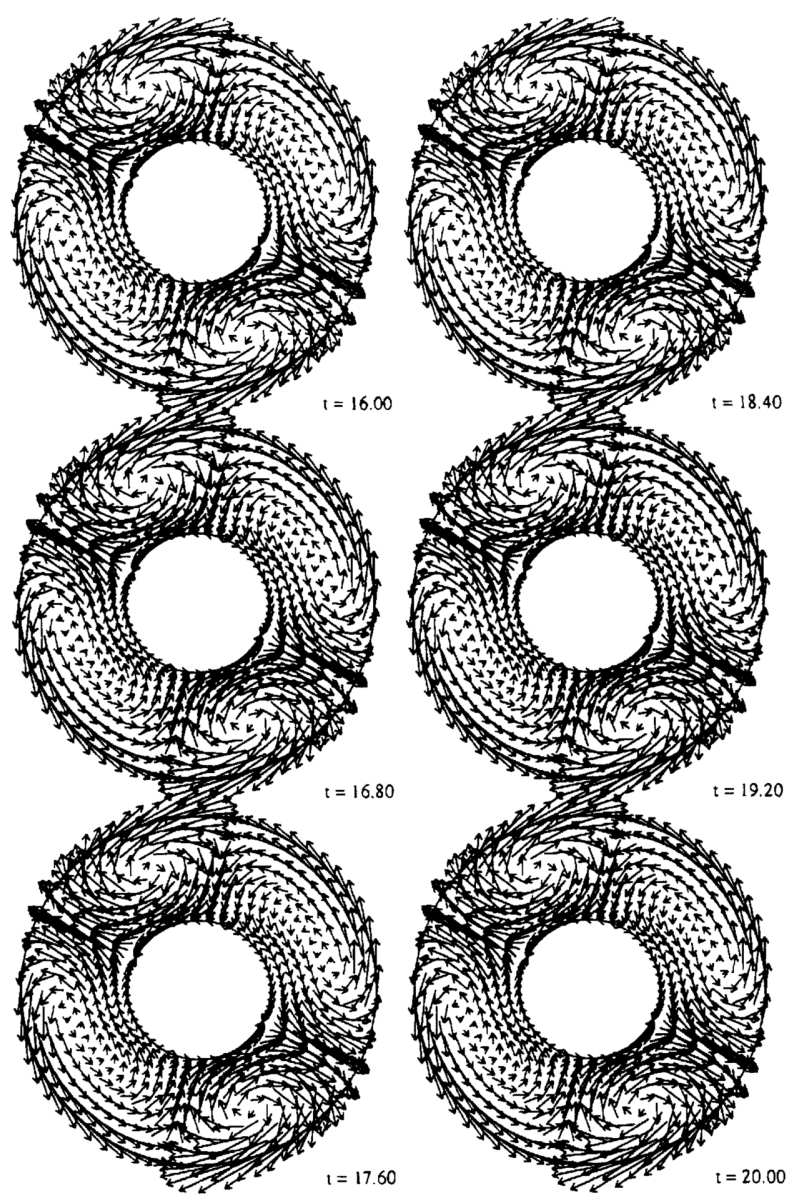

Fig. 4. Pattern of convective motion in the spherical shell. Arrows indicate the velocity field on the equatorial plane viewed from the north pole side. The origin of the longitude is taken at the due right position in each diagram.

(ZHANG and GubBins, 1992). The static feature would be a result of competition between the pattern of temperature variation at the outer shell and the drift of convection cells occurring naturally without the temperature variation, and the phase of the static cells reflects the balance between these two. Such a feature is more clearly shown in ZHANG and GuBBINS (1993).

Another point to be noted is that the magnitude of velocity field is enhanced, compared with the homogeneous case. The shift of the upwellings is also recognized in Fig. 5 showing the velocity field near the outer boundary. If this figure is compared with Fig. 3, we find that the fluid rises between the hot and cold areas at the outer boundary and moves toward the cold areas.

Time evolution of the velocity and the magnetic fields is shown in terms of their energy in Fig. 6 for dominant constituents. All the fields evolve from initial values to stationary values with some fluctuations at an early stage. In this case, energy exchanges between the velocity and the magnetic fields are supposed to be balanced. The Gauss coefficients no longer change their signs. This can be regarded as a steady dynamo model. 

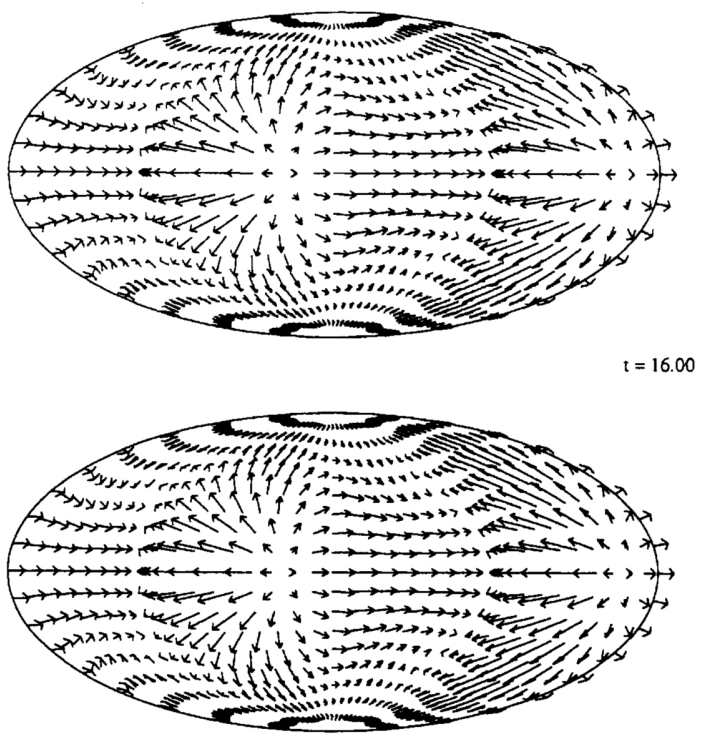

$t=16.80$

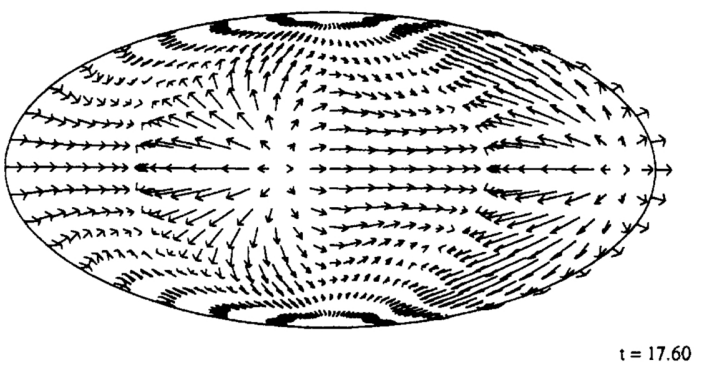

Fig. 5. Pattern of convective motion near the outer boundary of the spherical shell. This pattern should be compared with the temperature distribution shown in Fig. 3.

4. Differences in Magnetic Field Behavior between Homogeneous and Inhomogeneous Models

An MHD dynamo system is expected to be very unstable and behave chaotically. This is the reason why we have examined models with dimensionless values slightly above the critical ones for which the magnetic field can be sustained. This strategy, however, resulted in different Prandtl numbers; that is, 30 for the homogeneous model in Section 2 and 10 for the inhomogeneous model in Section 3. We should remember here that the main purpose of this paper has been to examine the effect of $\mathrm{CMB}$ on polarity reversals of the magnetic field. Then the dimensionless numbers describing the models should be the same except for variations of the temperature at the outer boundary. In this sense, direct comparison of the magnetic field behavior for the inhomogeneous model in Section 3 with that for the homogeneous model in Section 2 may not make sense.

On the other hand, if model parameters are far above the critical ones, the magnetic field would undergoes very irregular fluctuations and no systematic pattern would be recognized unless very extensive studies are made. Since the dynamical behavior turned out to be more stable than the magnetic field behavior, we fixed the magnetic Prandtl number at 10 and increased the Rayleigh number slightly so that the magnetic field is sustained against the ohmic dissipation; the magnetic field cannot be sustained for the model in Section 2, if the magnetic Prandtl number 


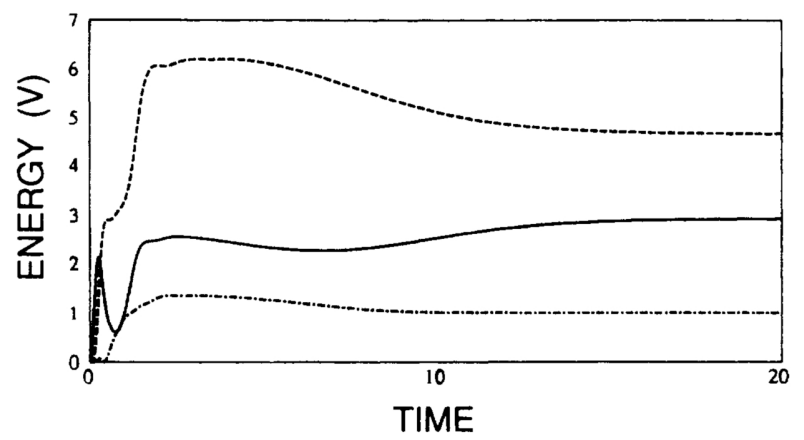

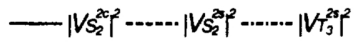
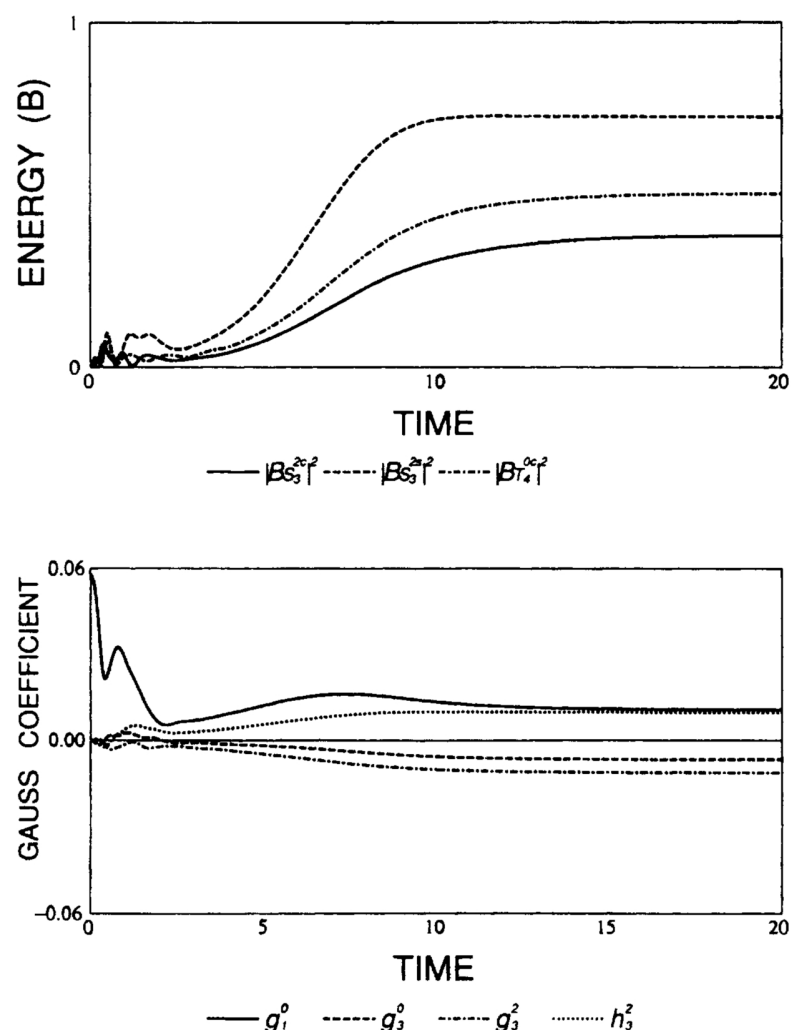

Fig. 6. Time evolution of the dominant constituents of the velocity and the magnetic fields in terms of their energy, and time evolution of the Gauss coefficients. Inhomogeneous model $(\operatorname{Ra}=900, \operatorname{Pr}=1, T a=100$, $P m=10, A=0.03$ ). 


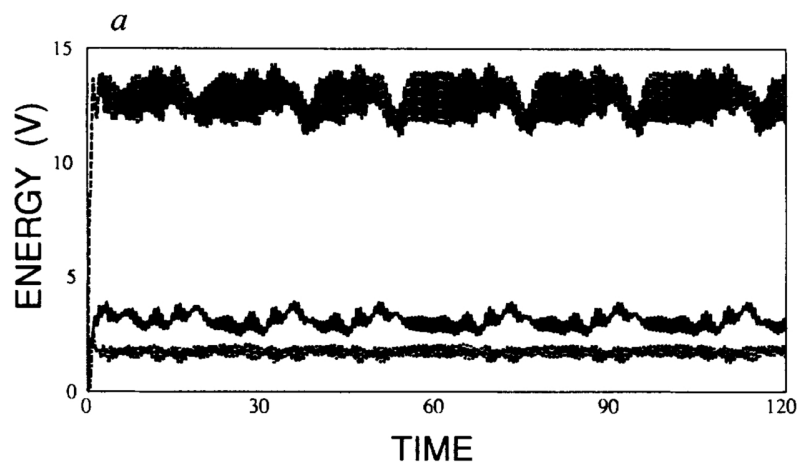

$-\left|V s_{2}^{0}\right|^{2}-\cdots\left|V s_{2}^{2}\right|^{2}-\cdots-\cdots|-| V T_{3}^{22}$

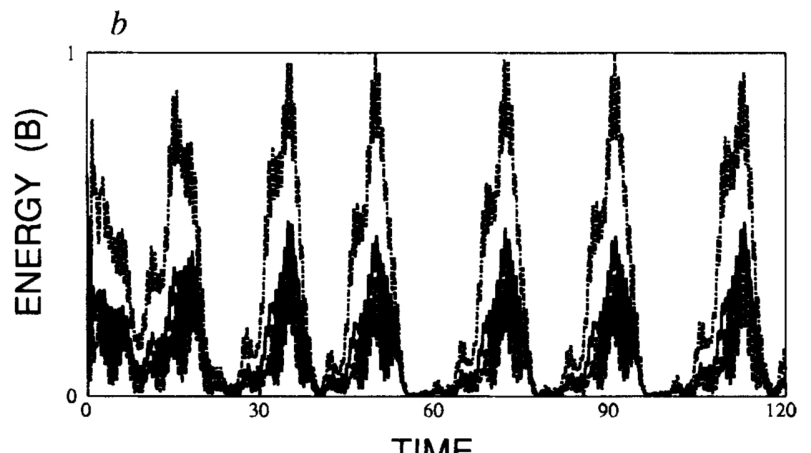

$\left|B s_{1}^{0}\right|^{2}-\cdots\left|B s_{3}^{0}\right|^{2} \cdots-\left.\cdots s_{3}^{2}\right|^{2}$

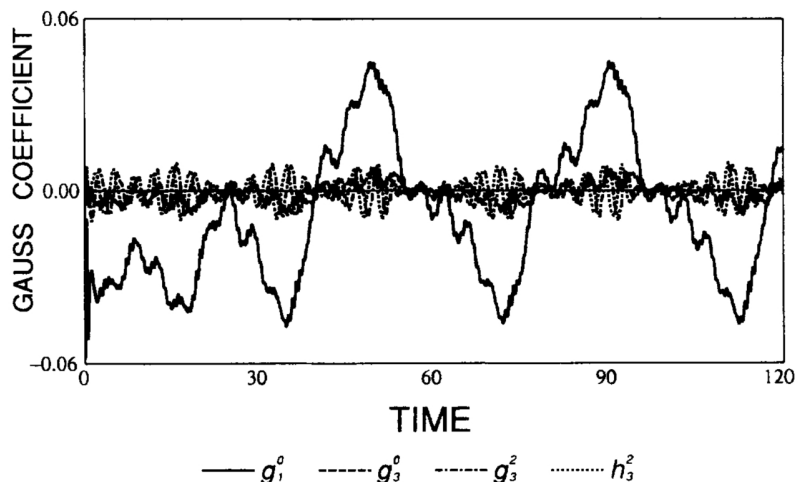

Fig. 7. Time evolution of the dominant constituents of the velocity and the magnetic fields in terms of their energy, and time evolution of the Gauss coefficients. Homogeneous model $(R a=1320, \operatorname{Pr}=1, T a=100$, $P m=10)$. 

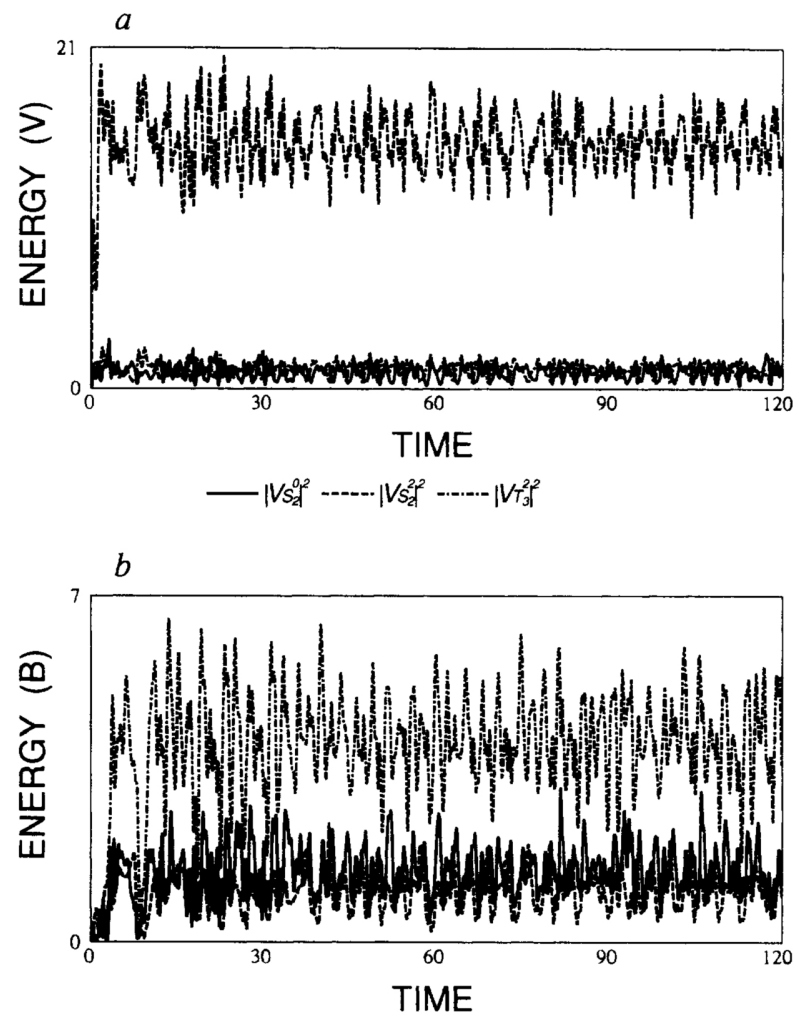

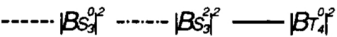

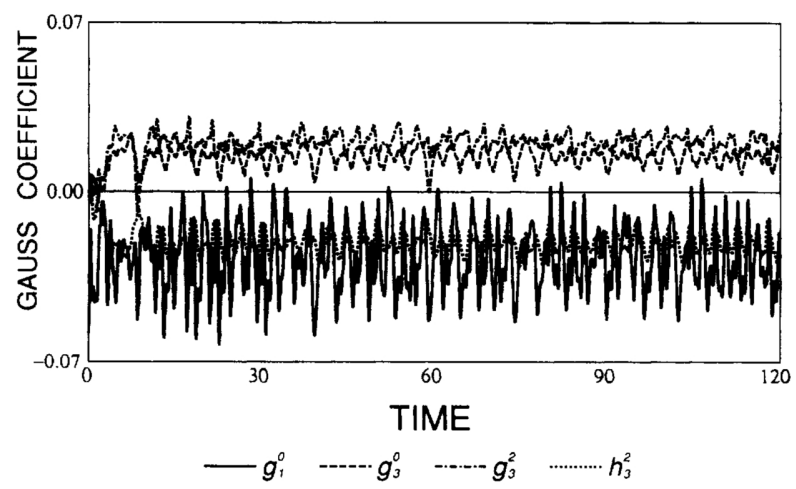

Fig. 8. Time evolution of the dominant constituents of the velocity and the magnetic fields in terms of their energy, and time evolution of the Gauss coefficients. Inhomogeneous model $(R a=1320, \operatorname{Pr}=1, T a=100$, $P m=10, A=0.03)$. 

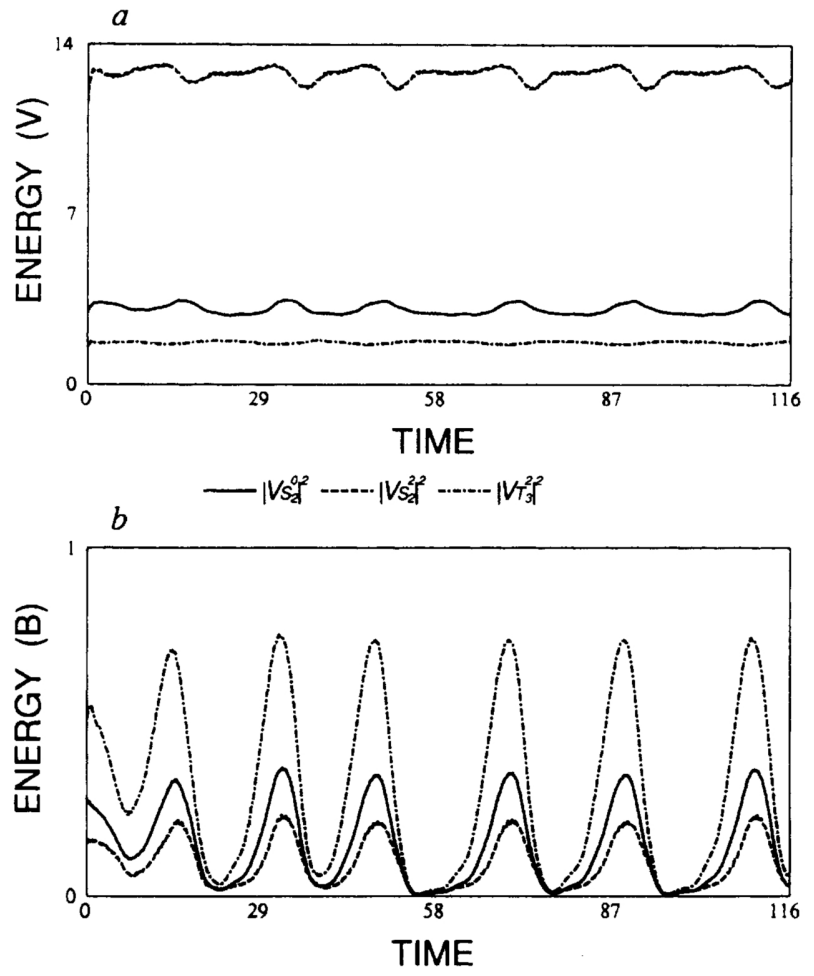

$-\left|B s_{1}^{0}\right|^{2} \cdots\left|B s_{j}^{0}\right|^{2} \cdots\left|-s_{s}^{2}\right|^{2}$

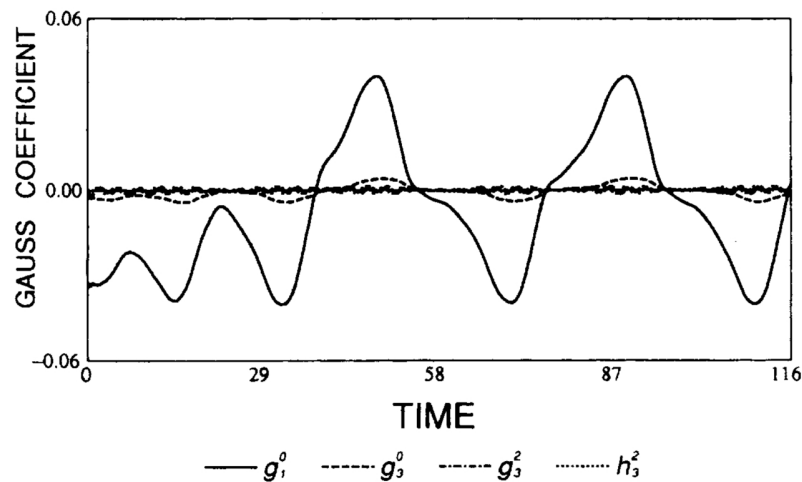

Fig. 9. Moving averages, over 4 dimensionless time units, of time variations of the velocity and magnetic field energy and the Gauss coefficients shown in Fig. 7. 


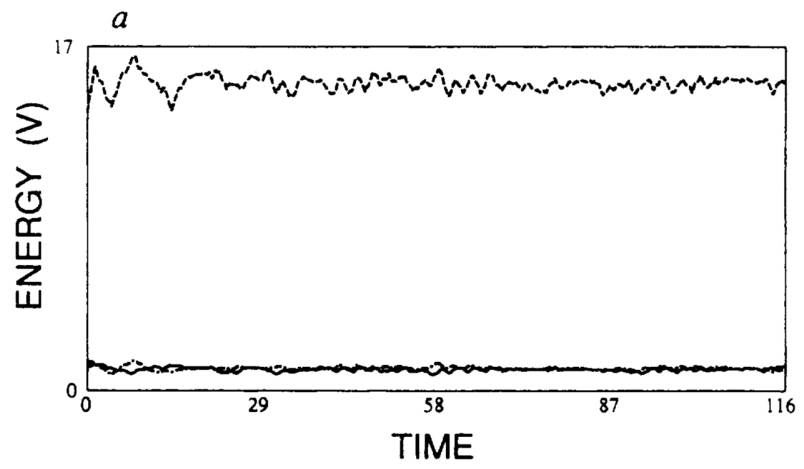

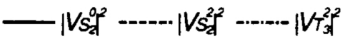

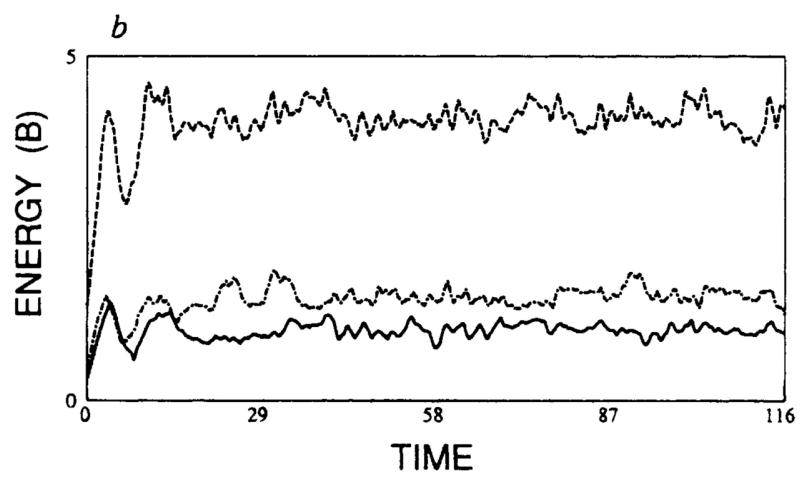

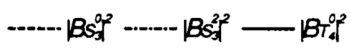

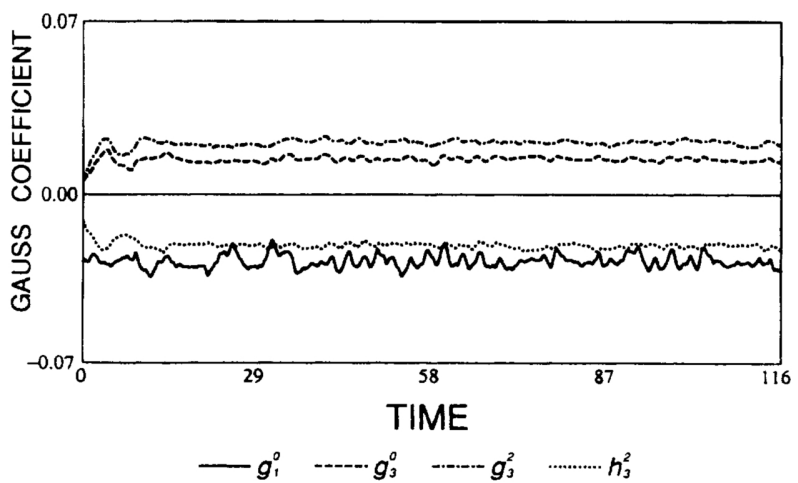

Fig. 10. Moving averages, over 4 dimensionless time units, of time variations of the velocity and magnetic field energy and the Gauss coefficients shown in Fig. 8. 
is 10 .

We now show the results for $R a=1320$ and $P m=10(P r=1, T a=100)$. The amplitude of temperature variation $A$ is again taken as 0.03 , the same as that for the previous inhomogeneous model. Figure 7 shows the result for the homogeneous model. In this case, the velocity and magnetic fields fluctuate considerably, but we can also recognize long-term variations; in particular, the dipole magnetic field represented by the $g_{1}^{0}$-term changes its polarity.

Such short-term fluctuations are more marked in the inhomogeneous case, as shown in Fig. 8 . Especially, the $g_{1}^{0}$-term, fluctuates around a mean negative value, and it sometimes takes a positive value. Such a variation is not a pure polarity reversal but rather an "excursion". In sedimentary paleomagnetic records, from which transitional magnetic field behavior can be inferred, the magnetic field is somehow smoothed over a certain time window (e.g. HYoDO, 1984) and here we simply took a moving average over 4 dimensionless time units, as shown in Figs. 9 and 10. The dipole magnetic field reverses its polarity in the homogeneous case, whereas it stays at a negative polarity with irregular short-term fluctuations.

\section{Discussion and Concluding Remarks}

Various mechanisms have been proposed for polarity reversals of the Earth's magnetic field. They are classified largely into the two categories. One requires a specific condition for triggering reversals; in particular, processes through the CMB have attracted much attention (e.g. GuBBINS, 1987). The other requires no specific conditions on the contrary and reversals have been considered rather as part of a dynamo process; that is, reversals occur spontaneously. The most typical example is the Rikitake coupled disk dynamo model (Rikitake, 1958).

In this respect, our homogeneous model suggests the latter mechanism as demonstrated in Section 2. The example shown there corresponds to the case in which dimensionless parameter values are slightly above the critical ones for instability to occur. It would be expected that if the parameter values are increased, more chaotic behavior would prevail, but the basic property would be little affected; that is, reversals can occur. This is in fact what we see in Section 4 . On the other hand, in our inhomogeneous model, the pattern of convective motion tends to stay stationary and no polarity reversal of the magnetic field occurs any longer, as was demonstrated clearly in Section 3 and with some modifications in Section 4.

As far as the present homogeneous and inhomogeneous models are concerned, the effect of the CMB seems to be very significant and even control the frequency of polarity reversal. In fact, if the amplitude of lateral temperature variation is taken between 0 and 0.03 , a steady drift of convection cells is gradually perturbed as the amplitude increases. For a small amplitude of temperature variation, the drift velocity fluctuates depending on the phase of convection cells. As the amplitude further increases, such a tendency becomes more marked and eventually the drift comes to a complete halt. According to these dynamical changes, polarity reversals become less frequent and no reversals occur, at least in a long time scale, as the drift stops.

Since the time constant for thermal processes in the mantle is much longer than that in the core, the long-term trend in reversal frequency is likely to be controlled by the mantle, particularly by the $\mathrm{D}^{\prime \prime}$ layer. In this respect, the exceptionally long normal epoch in Cretaceous has been related to extensive mantle plumes (LARSON, 1991a, b; LARSON and OLSON, 1991). Based on our models, we can now understand this as implying a perturbed and hence inhomogeneous thermal state at the base of the mantle where thermal plumes are supposed to originate, although the thermal effect of the $\mathrm{D}^{\prime \prime}$ layer may not be so simple as pointed out by LOPER (1992).

This speculation, however, should be evaluated critically, since we have examined only a few models selected among numerous relevant models even if we restrict parameter values to nearly critical ones. Moreover, the effects of truncation at various levels have not been explored yet and no general conclusion can be derived at present. This problem may turn out to be crucial if 
further progress is made towards a model which is relevant to the real Earth. Also, the discussion should be based on the degree of inhomogeneity in radial temperature gradient at the CMB. In this respect, we have just started calculations for conditions of temperature gradient at the outer boundary of the spherical shell.

Finally, we emphasize the aim of this paper. So far, polarity reversals have been discussed on the basis of simple coupled-disk dynamo models or speculative arguments on magnetohydrodynamics in the core. Our models are more realistic than coupled-disk dynamo models even if our models are still far from the real Earth. Moreover, the discussion is based on numerical calculations and hence quantitative. We believe that further progress should be made along such a line.

We are thankful to Dave Loper for his comments on our work during the SEDI Mizusawa symposium. We also thank Keke Zhang and an anonymous referee for their valuable comments. This work was supported by the Ministry of Education, Science and Culture under grant-in-aid for scientific research on priority area No. 04216105. Contribution of the Department of Earth and Planetary Sciences, Tokyo Institute of Technology, No. 13.

\section{REFERENCES}

Allan, D. W., On the behaviour of systems of coupled dynamos, Proc. Camb. Phil. Soc., 58, 671-693, 1962.

Bloxham, J. and D. Glbbins, Thermal core-mantle interactions, Nature, 325, 511-513, 1987.

Bloxham, J. and A. JACKsON, Lateral temperature variations at the core-mantle boundary deduced from the magnetic field, Geophys. Res. Lett., 17, 1997-2000, 1990.

GubBins, D., Mechanism for geomagnetic polarity reversals, Nature, 326, 167-169, 1987.

Gubbins, D., Thermal core-mantle interactions and time-averaged paleomagnetic field, J. Geophys. Res., 93, 3413-3420, 1988.

Hoffman, K. A., Dipolar reversal states of the geomagnetic field and core-mantle dynamics, Nature, 359, 789-794, 1992.

Honkura, Y. and M. Matsushima, Fluctuation of the nondipole magnetic field and its implication for the process of geomagnetic polarity reversal in the Cox model, J. Geophys. Res., 93, 11,631-11,642, 1988.

Honkura, Y., T. Iijima, and M. Matsushima, Magnetic field reversal resulting from a dynamo process in a spherical shell, J. Geomag. Geoelectr., 44, 931-941, 1992.

Hyodo, M., Possibility of reconstruction of the past geomagnetic field from homogeneous sediments, J. Geomag. Geoelectr., 36, 45-62, 1984.

Jones, G. M., Thermal interaction of the core and the mantle and long-term behavior of the geomagnetic field, $J$. Geophys. Res., 83, 1703-1709, 1977.

Laj, C., A. Mazaud, R. Weeks, M. Fuller, and E. Herrero-Bervera, Statistical assessment of the preferred longitudinal bands for recent geomagnetic reversal records, Geophys. Res. Lett., 19, 2003-2006, 1992.

LARSON, R. L., Latest pulse of Earth: Evidence for a mid-Cretaceous superplume, Geology, 19, 547-550, 1991a.

LARSON, R. L., Geological consequences of superplumes, Geology, 19, 963-966, $1991 \mathrm{~b}$.

Larson, R. L. and P. Olson, Mantle plumes control magnetic reversal frequency, Earth Planet. Sci. Lett., 107, 437-447, 1991.

LOPER, D. E., On the correlation between mantle plume flux and the frequency of reversals of the geomagnetic field, Geophys. Res. Lett., 19, 25-28, 1992.

Olson, P. and V. L. HAGEe, Geomagnetic polarity reversals, transition field structure, and convection in the outer core, J. Geophys. Res., 95, 4609-4620, 1990.

Rikitake, T., Oscillations of a system of disk dynamos, Proc. Camb. Phil. Soc., 54, 89-105, 1958.

Shimizc, M. and Y. HonkURA, Statistical nature of polarity reversals of the magnetic field in coupled-disk dynamo models, J. Geomag. Geoelectr., 37, 455-497, 1985.

ZhANG, K.-K. and F. H. Busse, On the onset of convection in rotating spherical shells, Geophys. Astrophys. Fluid Dynamics, 39, 119-147, 1987.

ZhANG, K. and D. GuBBINS, On convection in the earth's core driven by lateral temperature variations in the lower mantle, Geophys. J. Int., 108, 247-255, 1992.

ZhANG, K. and D. GubBins, Convection in a rotating spherical fluid shell with an inhomogeneous temperature boundary condition at infinite Prandtl number, J. Fluid Mech., 250, 209-232, 1993. 